\title{
Orthorectified Polar Format Algorithm for Generalized Spotlight SAR Imaging With DEM
}

\author{
Ruizhi $\mathrm{Hu}^{\circledR}$, Member, IEEE, Bhavani Shankar Mysore Rama Rao ${ }^{\circledR}$, Senior Member, IEEE, \\ Mohammad Alaee-Kerahroodi ${ }^{\circledR}$, Member, IEEE, and Björn Ottersten $^{\circledR}$, Fellow, IEEE
}

\begin{abstract}
In conventional polar format algorithm (PFA), the effective imaging scene is bounded to a limited region near the reference point unless postprocessing is utilized. In a previous paper, refocusing and zoom-in PFA (RZPFA) for curvilinear spotlight SAR imaging were proposed to produce a refocused image for an arbitrary region of interest (ROI) with constant elevation. However, for certain applications, the residual distortion and defocus caused by rugged terrain could not be ignored. In this article, RZPFA is adapted to incorporate the known digital elevation model (DEM) into the imaging process, which is named as orthorectified PFA (OPFA). With just little additional computations than RZPFA, OPFA can realize georeferenced orthorectified imaging via a nonuniform fast Fourier transform of type 3 (NuFFT-3) without the need of postprocessing. The quantitative metrics for the residual DEM distortion and residual DEM defocus were also derived to determine the effective imaging extent of OPFA. Within the effective extent, the proposed OPFA can obtain an orthorectified image efficiently, and the image has a very high quality comparable to backprojection (BP). The imaging results of measured echo and DEM data demonstrated the effectiveness of the proposed algorithm.
\end{abstract}

Index Terms-Digital elevation model (DEM), georeferenced imaging, nonuniform fast Fourier transform, orthorectified synthetic aperture radar (SAR) imaging, polar format algorithm (PFA).

\section{INTRODUCTION}

$\mathbf{T}$ HE past decades have witnessed an ever-increasing development in the fields of remote sensing [1], among which synthetic aperture radar (SAR) is of great importance for its unique all-day, all-weather sensing capability [2]. Similar to many other fields, the research of SAR has also entered the era of image processing based on deep learning [3], and commercial SAR startups are springing up [4], [5]. The research in SAR imaging has focused on algorithms for novel modes, such as bistatic SAR [6], multistatic SAR [7], multiple input multiple output SAR (MIMO-SAR) [8], and video

Manuscript received June 5, 2020; revised July 8, 2020; accepted July 18, 2020. This work was supported by the Luxembourg National Research Fund (FNR) through the CORE project "SPRINGER" under Grant C18/IS/12734677/SPRINGER. (Corresponding author: Ruizhi Hu.)

The authors are with the Interdisciplinary Centre for Security, Reliability and Trust (SnT), University of Luxembourg, L1885 Luxembourg City, Luxembourg (e-mail: ruizhi.hu@uni.lu; bhavani.shankar@uni.lu; mohammad.alaee@ uni.lu; bjorn.ottersten@uni.lu).

Color versions of one or more of the figures in this article are available online at http://ieeexplore.ieee.org.

Digital Object Identifier 10.1109/TGRS.2020.3011638
SAR [9], [10], as well as imaging of moving targets [11] and computational imaging algorithms with constraints [12].

Most conventional SAR imaging algorithms rely on the assumption of flat terrain to enable easier representation in the frequency domain. Departing from this assumption, the backprojection (BP) algorithm accommodates a rugged scenario by virtue of its operation in time-domain, where the digital elevation model (DEM) can be easily incorporated into the calculation of slant ranges. Known for its high efficiency and simple implementation, with proper modifications shown in this article, the polar format algorithm (PFA) [13] can also form orthorectified image directly. As a result, the traditional orthorectification process [14], [15] becomes unnecessary for PFA, and the imaging results can be directly fused with orthoimageries obtained from other sensors.

Conventionally, BP has three advantages over PFA: 1) inherent accommodation for curved wavefronts without geometric distortions or image defocus; 2) feasibility of imaging of echo data collected on an arbitrary flight path; and 3) direct computation of an orthorectified image onto an existing DEM without depth-of-focus distortions [16]. The first two advantages of BP mentioned above have also been realized by the refocusing and zoom-in polar format algorithm (RZPFA) [17], which is efficiently realized via a nonuniform fast Fourier transform of type 3 (NuFFT-3) [18], [19]. Building on the RZPFA, in this article, we propose another variant of PFA, namely, orthorectified PFA (OPFA), which shows that DEM can also be incorporated in PFA and result in orthorectified imaging both accurately and efficiently.

The rest of this article is organized as follows. In Section II, the geometry of imaging on nonflat scenario and the signal model is presented. In Section III, OPFA is explained in detail. In Section IV, some commentaries on OPFA and its connections with RZPFA are given. In Section V, experimental results on measured echo and DEM data are provided. In Section VI, conclusions are given.

\section{IMAGing GeOMEtRy AND Signal Model}

The imaging geometry on a nonflat terrain is shown in Fig. 1. There are two coordinate systems: one is the original coordinate system (OCS) defined by the motion measurements $(\mathbf{0}-x y z$, black colored) and the other one is the refocusing coordinate system $(\mathrm{ReCS})$ centered at the region 


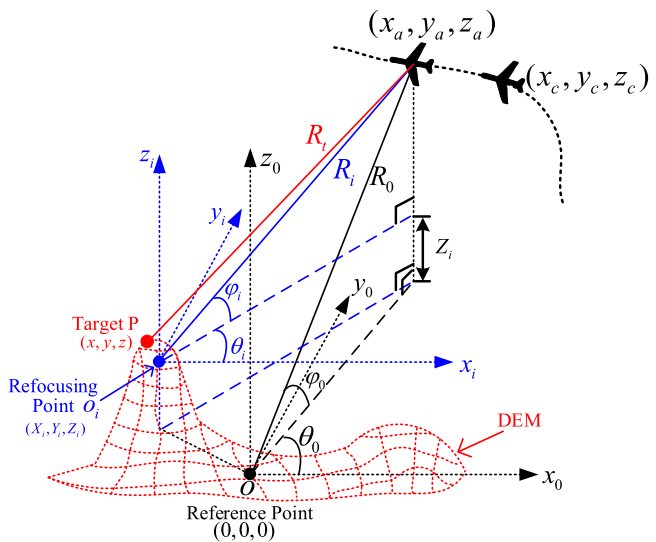

Fig. 1. Geometry of curvilinear spotlight SAR imaging with DEM.

of interest (ROI, $\mathbf{o}_{i}-x_{i} y_{i} z_{i}$, blue colored). The radar-carrying platform flies along a general curvilinear trajectory and works in spotlight mode. Let $\mathbf{r}_{\mathbf{a}}=\left[x_{a}, y_{a}, z_{a}\right]^{T}$ denote the instantaneous location of radar antenna phase center (APC), then its instantaneous slant range from $\mathbf{o}$ is given by

$$
R_{0}=R_{0}(t)=\left\|\mathbf{r}_{\mathbf{a}}\right\|=\sqrt{x_{a}^{2}+y_{a}^{2}+z_{a}^{2}} .
$$

Let $\mathbf{r}_{\mathbf{t}}=[x, y, z(x, y)]^{T}$ denote the location of an arbitrary target $P$ in OCS, with the elevation value given by the known DEM $z(x, y)$, then its instantaneous slant range from $\mathbf{r}_{\mathbf{a}}$ is

$$
\begin{aligned}
R_{t} & =R_{t}(x, y, z(x, y) ; t)=\left\|\mathbf{r}_{\mathbf{t}}-\mathbf{r}_{\mathbf{a}}\right\| \\
& =\sqrt{\left(x-x_{a}\right)^{2}+\left(y-y_{a}\right)^{2}+\left(z-z_{a}\right)^{2}} .
\end{aligned}
$$

To simplify subsequent analysis without the loss of generality, the curvilinear synthetic aperture is assumed to be occurred in the time interval of $t \in[-1,1]$, and the time instant at the aperture center is defined as the initial time $(t=0)$. Let $\mathbf{r}_{\mathbf{c}}=\left[x_{c}, y_{c}, z_{c}\right]^{T}$ denote the coordinates of the synthetic aperture center, its slant range from $\mathbf{r}_{\mathbf{t}}$ is written as

$$
\begin{aligned}
R_{t c} & =R_{t}(x, y, z(x, y) ; t=0)=\left\|\mathbf{r}_{\mathbf{t}}-\mathbf{r}_{\mathbf{c}}\right\| \\
& =\sqrt{\left(x-x_{c}\right)^{2}+\left(y-y_{c}\right)^{2}+\left(z-z_{c}\right)^{2}} .
\end{aligned}
$$

After dechirping and range de-skew [20], the phase of echoed signal from target $P$ can be expressed as

$$
S_{0}=\exp \left[-j K\left(R_{t}-R_{0}\right)\right]=\exp \left(-j K \Delta R_{0}\right)
$$

where $K=4 \pi f / c$ denotes the wavenumber, $f$ is the linear frequency, $c$ is the speed of light, and $\Delta R_{0}=R_{t}-R_{0}$ denotes the differential range of $P$ with respect to $\mathbf{0}$.

\section{OPFA Methodology}

OPFA comprises of four main procedures: 1) a refocusing process to reset the ROI center as the refocusing point; 2) calculation of the nonuniform input wavenumber distribution after refocusing; 3) calculation of the nonuniform output pixel grid based on the derived orthorectified distortion mapping (ODM); and 4) orthorectified imaging via NuFFT-3. The methodology of OPFA is very similar to RZPFA [17], and the only difference is that OPFA is more general, where nonflat scene is considered and DEM is incorporated, so that orthorectified image can be obtained directly. In short, OPFA has all the advantages of $\mathrm{BP}$, yet it still keeps the high efficiency of the PFA family [17], [21], [22].

\section{A. Refocusing}

As demonstrated in [17] and [20]-[22], the plane-wave assumption in PFA will lead to intolerable distortion and defocus when the target gets farther away from $\mathbf{o}$. In addition, when the scene is nonflat, targets' elevation offsets from the imaging plane will cause additional distortion and defocus.

Given the DEM of the ROI and accurate positional measurements of the radar platform, it is feasible to reset the ROI center as the new reference point $\mathbf{o}_{\mathbf{i}}$ via a simple phase compensation on $S_{0}$ [17]. After refocusing, the above two sources of distortion and defocus can be largely mitigated altogether. As a result, within a certain area around the ROI, image defocus can be neglected, and the extent for the defocusnegligible region for a nonflat terrain after refocusing will be given in Section IV-B.

Let $\mathbf{o}_{\mathbf{i}}=\left[X_{i}, Y_{i}, Z_{i}\right]^{T}$ denote the coordinates of ROI center in OCS. As colored blue in Fig. 1, the instantaneous slant range between $\mathbf{r}_{\mathbf{a}}$ and $\mathbf{o}_{\mathbf{i}}$ can be calculated by

$$
\begin{aligned}
R_{i} & =R_{t}\left(X_{i}, Y_{i}, Z_{i} ; t\right)=\left\|\mathbf{r}_{\mathbf{a}}-\mathbf{o}_{\mathbf{i}}\right\| \\
& =\sqrt{\left(X_{i}-x_{a}\right)^{2}+\left(Y_{i}-y_{a}\right)^{2}+\left(Z_{i}-z_{a}\right)^{2}}
\end{aligned}
$$

, and the slant range between $\mathbf{r}_{\mathbf{c}}$ and $\mathbf{o}_{\mathbf{i}}$ is given by

$$
\begin{aligned}
R_{i c} & =R_{t}\left(X_{i}, Y_{i}, Z_{i} ; t=0\right)=\left\|\mathbf{r}_{\mathbf{c}}-\mathbf{o}_{\mathbf{i}}\right\| \\
& =\sqrt{\left(X_{i}-x_{c}\right)^{2}+\left(Y_{i}-y_{c}\right)^{2}+\left(Z_{i}-z_{c}\right)^{2}} .
\end{aligned}
$$

The differential range of $P$ with respect to $\mathbf{o}_{\mathbf{i}}$ is written as

$\Delta R_{i}=R_{t}-R_{i}=\left(R_{t}-R_{0}\right)+\left(R_{0}-R_{i}\right)=\Delta R_{0}+\Delta_{i}$

where $\Delta_{i}=R_{0}-R_{i}$ is the instantaneous difference between $R_{0}$ and $R_{i}$.

Let $S_{i}$ denote the ideal dechirped signal phase from $P$ with respect to $\mathbf{o}_{\mathbf{i}}$. According to (4) and (7), then $S_{i}$ can be obtained via a simple phase compensation from $S_{0}$ as

$$
\begin{aligned}
S_{i} & =\exp \left(-j K \Delta R_{i}\right)=\exp \left[-j K\left(\Delta R_{0}+\Delta_{i}\right)\right] \\
& =S_{0} \exp \left(-j K \Delta_{i}\right) .
\end{aligned}
$$

\section{B. Wavenumber Distribution After Refocusing}

When the imaging scene is small enough compared to its distance from the radar APC, after refocusing on $\mathbf{o}_{\mathbf{i}}$, followed by a first-order 2-D spatial Taylor series expansion of $\Delta R_{i}$ with respect to $(x, y)=\left(X_{i}, Y_{i}\right)$, the approximate differential range of PFA under plane-wave assumption is given by [17], [21]:

$$
\Delta R_{p i}=-\frac{\left(x_{a}-X_{i}\right)\left(x-X_{i}\right)+\left(y_{a}-Y_{i}\right)\left(y-Y_{i}\right)}{R_{i}}
$$

where $\left(x-X_{i}, y-Y_{i}\right)$ is the distortion-free reconstructed location of target $P$ in the refocused PFA image centered at $\mathbf{o}_{\mathbf{i}}$ on the imaging plane $x_{i}-\mathbf{o}_{\mathbf{i}}-y_{i}$. 
Let $\left(\hat{x}_{i}, \hat{y}_{i}\right)=\left[\hat{x}_{i}(x, y ; z(x, y)), \hat{y}_{i}(x, y ; z(x, y))\right]$ denote the actual reconstructed location of target $P$ in the refocused PFA image. Because of an increasing extent or a drastic elevation variation of the ROI, distortion may become nonnegligible even after the refocusing, which means $\left(\hat{x}_{i}, \hat{y}_{i}\right) \neq$ $\left(x-X_{i}, y-Y_{i}\right)$. In this case, $\Delta R_{p i}$ should be rewritten as

$$
\Delta R_{p i}=-\frac{\left(x_{a}-X_{i}\right) \hat{x}_{i}+\left(y_{a}-Y_{i}\right) \hat{y}_{i}}{R_{i}} .
$$

Fortunately, the 2.5-D-to-2-D mapping of $(x, y ; z(x, y)) \rightarrow$ $\left(\hat{x}_{i}, \hat{y}_{i}\right)$, namely ODM, can be derived and will be given later in Section III-C.

In (8), by replacing $\Delta R_{i}$ with $\Delta R_{p i}$ given in (10), $S_{i}$ is now approximated as

$$
\begin{aligned}
S_{i} & \approx \exp \left(-j K \Delta R_{p i}\right)=\exp \left[j K \frac{\left(x_{a}-X_{i}\right) \hat{x}_{i}+\left(y_{a}-Y_{i}\right) \hat{y}_{i}}{R_{i}}\right] \\
& =\exp \left(j \hat{K}_{x i} \hat{x}_{i}+j \hat{K}_{y i} \hat{y}_{i}\right)
\end{aligned}
$$

where $\hat{K}_{x i}$ and $\hat{K}_{y i}$ are the Cartesian wavenumber in ReCS, given by

$$
\hat{K}_{x i}=K \cos \varphi_{i} \cos \theta_{i}, \quad \hat{K}_{y i}=K \cos \varphi_{i} \sin \theta_{i}
$$

and $\theta_{i}$ and $\varphi_{i}$ are the azimuth and depression angle of $\mathbf{r}_{\mathbf{a}}$ with respect to $\mathbf{o}_{\mathbf{i}}$, which are shown in Fig. 1 and are expressed as

$$
\varphi_{i}=\arcsin \left(\frac{z_{a}-Z_{i}}{R_{i}}\right), \quad \theta_{i}=\arctan \left(\frac{y_{a}-Y_{i}}{x_{a}-X_{i}}\right) .
$$

From (12) and (13), it is shown that the wavenumber is still in polar format, and its 2-D distribution of $\left(\hat{K}_{x i}, \hat{K}_{y i}\right)$ is nonuniform and depends on $\mathbf{0}_{\mathbf{i}}$.

\section{C. $O D M$}

When the extent of the ROI is relatively large or the image resolution is relatively high, the plane-wave assumption will fail, and the problem of residual distortion after refocusing should be considered and compensated [17]. Particularly, the elevation variation of a nonflat scene will further aggravate the distortion effect. To describe the image distortion pattern in the refocused PFA image of a nonflat scene, ODM is proposed and derived. ODM includes both the original distortion of PFA for a flat terrain and the additional distortion caused by a nonflat terrain (DEM distortion).

The location of a target in the reconstructed SAR image is determined by the constant and first-order temporal terms of the differential range. Similar to [17], [21], and [22], after temporal Taylor series expansion of $\Delta R_{i}$ and $\Delta R_{p i}$ at the aperture center $(t=0)$, followed by equating their respective constant and first-order temporal coefficients, ODM can be derived as:

$$
\begin{aligned}
& \hat{x}_{i}=\hat{x}_{i}(x, y ; z(x, y))=\frac{\dot{y}_{c} D_{i}-\left(y_{c}-Y_{i}\right) E_{i}}{F_{i}} \\
& \hat{y}_{i}=\hat{y}_{i}(x, y ; z(x, y))=\frac{-\dot{x}_{c} D_{i}+\left(x_{c}-X_{i}\right) E_{i}}{F_{i}}
\end{aligned}
$$

where $\left(\dot{x}_{c}, \dot{y}_{c}, \dot{z}_{c}\right)$ denotes the first-order temporal derivatives of $\left(x_{a}, y_{a}, z_{a}\right)$ at $t=0$, and $D_{i}, E_{i}, F_{i}, A_{i}$, and $A$ are intermediate mapping functions defined as

$$
\begin{aligned}
D_{i} & =D_{i}(x, y ; z(x, y))=R_{i c}^{2}-R_{i c} R_{t c} \\
E_{i} & =E_{i}(x, y ; z(x, y))=2 A_{i}-\frac{A R_{i c}}{R_{t c}}-\frac{A_{i} R_{t c}}{R_{i c}} \\
F_{i} & =\left(x_{c}-X_{i}\right) \dot{y}_{c}-\left(y_{c}-Y_{i}\right) \dot{x}_{c} \\
A & =A(x, y ; z(x, y)) \\
& =\left(x_{c}-x\right) \dot{x}_{c}+\left(y_{c}-y\right) \dot{y}_{c}+\left(z_{c}-z\right) \dot{z}_{c} \\
A_{i} & =\left(x_{c}-X_{i}\right) \dot{x}_{c}+\left(y_{c}-Y_{i}\right) \dot{y}_{c}+\left(z_{c}-Z_{i}\right) \dot{z}_{c} .
\end{aligned}
$$

The derivation of ODM is very similar to the refocused distortion mapping (RDM) in [17, Appendix A], except that RDM only considers the distortion of a flat scene, so we do not repeat here. To facilitate an easier reference, this article follows the same notations.

\section{OPFA Imaging via NuFFT-3}

Let $z_{r}=z\left(x_{r}, y_{r}\right)$ denote the known DEM defined on a uniform mesh grid $\left(x_{r}, y_{r}\right)$ that represents the ROI, and $\left(\hat{x}_{r i}, \hat{y}_{r i}\right)$ denote the corresponding distorted 2-D nonuniform grid. Given the sufficient accuracy of ODM, for each pixel on the DEM surface $\left(x_{r}, y_{r} ; z_{r}\right)$, its reconstructed location in the distorted refocused image can be calculated explicitly via ODM (14). Furthermore, as the ODM is a pixel-to-pixel mapping, through a direct calculation of the 2-D Fourier transform on the distorted nonuniform grid $\left(\hat{x}_{r i}, \hat{y}_{r i}\right)$, the intensity of dislocated pixels is stored back to their actual locations and image distortion is corrected simultaneously, and the process is represented by

$$
\begin{aligned}
I\left(x_{r}, y_{r}\right) \stackrel{\mathrm{ODM}}{=} & I_{i}\left(\hat{x}_{r i}, \hat{y}_{r i}\right) \\
= & \iint S_{i}\left(\hat{K}_{x i}, \hat{K}_{y i}\right) \\
& \times \exp \left(-j \hat{K}_{x i} \hat{x}_{r i}-j \hat{K}_{y i} \hat{y}_{r i}\right) d K_{x i} d K_{y i}
\end{aligned}
$$

where $I\left(x_{r}, y_{r}\right)$ denotes the desired orthorectified image, $I_{i}\left(\hat{x}_{r i}, \hat{y}_{r i}\right)$ denotes the distorted refocused PFA image, $\stackrel{\text { ODM }}{=}$ denotes pixelwise intensity equality between $I\left(x_{r}, y_{r}\right)$, and $I_{i}\left(\hat{x}_{r i}, \hat{y}_{r i}\right)$ via ODM, $\left(K_{x i}, K_{y i}\right)$ denotes the uniform Cartesian wavenumber grid in ReCS.

In (16), taking $\left(\hat{K}_{x i}, \hat{K}_{y i}\right)$ as the input grid and $\left(\hat{x}_{r i}, \hat{y}_{r i}\right)$ as the output grid, then both the input signal $S_{i}\left(\hat{K}_{x i}, \hat{K}_{y i}\right)$ and the output image $I_{i}\left(\hat{x}_{i}, \hat{y}_{i}\right)$ are nonuniformly distributed. Therefore, (16) takes the form of nonuniform Fourier transform of type-3 [18], [19]. As a result, the whole orthorectified imaging of OPFA can be realized efficiently via a single NuFFT-3.

\section{COMMEnTARIES ON OPFA}

In this section, the consistency and difference between OPFA and RZPFA are given. Furthermore, for an analysis of the effective imaging extent of OPFA, the residual DEM distortion and residual DEM defocus after refocusing is derived. Finally, this section ends with a brief summary and outlook of OPFA. 


\section{A. Consistency and Difference Between OPFA and RZPFA}

Clearly, the methodology subsumes the idealized case of flat scene as a special case. Particularly, with $z=0$ and $Z_{i}=0$, all the equations mentioned reduce to the corresponding equations in [17]. It proves that RZPFA is a special case of OPFA, or equivalently, RDM is a special case of ODM. Based on (14), RDM can be written as

$$
\begin{aligned}
& \hat{x}_{i 0}=\hat{x}_{i}\left(x, y ; z=0, Z_{i}=0\right)=\frac{\dot{y}_{c} D_{i 0}-\left(y_{c}-Y_{i}\right) E_{i 0}}{F_{i}} \\
& \hat{y}_{i 0}=\hat{y}_{i}\left(x, y ; z=0, Z_{i}=0\right)=\frac{-\dot{x}_{c} D_{i 0}+\left(x_{c}-X_{i}\right) E_{i 0}}{F_{i}}
\end{aligned}
$$

where $D_{i 0}$ and $E_{i 0}$ denote the corresponding functions of $D_{i}$ and $E_{i}$ in (15) by imposing $z=0$ and $Z_{i}=0$ on all related functions and variables.

As will be demonstrated by the experimental results in Section V-C, when defocus can be ignored, OPFA is equivalent to BP with DEM, and RZPFA is equivalent to BP without DEM. Consequently, a metric for the residual distortion merely caused by the terrain (DEM distortion) can be represented by the relative displacement between RDM and ODM as

$$
\begin{aligned}
\delta_{i}(x, y) & =\delta_{i}\left(x, y ; z(x, y), X_{i}, Y_{i}, Z_{i}\right) \\
& =\sqrt{\left(\hat{x}_{i}-\hat{x}_{i 0}\right)^{2}+\left(\hat{y}_{i}-\hat{y}_{i 0}\right)^{2}} .
\end{aligned}
$$

\section{B. Defocus-Negligible Region (DeR)}

After OPFA, image distortion is compensated. However, as ODM only guarantees the equivalence between the corresponding temporal Taylor expansion coefficients of $\Delta R_{i}$ and $\Delta R_{p i}$ up to the first order, image defocus caused by the residual quadratic phase error (RQPE) will still impose restrictions on the effective extent of OPFA. Let $\Phi_{q i}$ define the approximated maximum RQPE after refocusing and $\lambda_{c}$ denote the carrier wavelength. Similar to [17], $\Phi_{q i}$ can be expressed as a function of $(x, y)$ as

$$
\begin{aligned}
\Phi_{q i} & =\Phi_{q i}\left(x, y ; z(x, y), X_{i}, Y_{i}, Z_{i}\right) \\
& =\frac{2 \pi}{\lambda_{c}}\left|\begin{array}{c}
\frac{G R_{t c}^{2}-A^{2}}{R_{i c}^{3}}-\frac{G_{i} R_{i c}^{2}-A_{i}^{2}}{R_{i c}^{3}}+\ldots \\
-\frac{B_{i}\left(R_{i c} \ddot{R}_{i c}-2 \dot{R}_{i c}^{2}\right)}{R_{i c}^{3}}-\frac{2 C_{i} \dot{R}_{i c}-H_{i} R_{i c}}{R_{i c}^{2}}
\end{array}\right|
\end{aligned}
$$

where $\dot{R}_{i c}$ and $\ddot{R}_{i c}$ are the first-order and second-order temporal derivative values of $R_{i}$ at $t=0$, given by

$$
\dot{R}_{i c}=\frac{A_{i}}{R_{i c}}, \quad \ddot{R}_{i c}=\frac{G_{i} R_{i c}^{2}-A_{i}^{2}}{R_{i c}^{3}} .
$$

$B_{i}, C_{i}$, and $H_{i}$ are the intermediate functions of $(x, y ; z(x, y))$ based on ODM

$$
\begin{aligned}
B_{i} & =B_{i}(x, y ; z(x, y))=\left(x_{c}-X_{i}\right) \hat{x}_{i}+\left(y_{c}-Y_{i}\right) \hat{y}_{i} \\
C_{i} & =C_{i}(x, y ; z(x, y))=\dot{x}_{c} \hat{x}_{i}+\dot{y}_{c} \hat{y}_{i} \\
H_{i} & =H_{i}(x, y ; z(x, y))=\ddot{x}_{c} \hat{x}_{i}+\ddot{y}_{c} \hat{y}_{i} .
\end{aligned}
$$

$G$ and $G_{i}$ are the intermediate functions given as

$$
\begin{aligned}
G= & G(x, y ; z(x, y)) \\
= & \dot{x}_{c}^{2}+\dot{y}_{c}^{2}+\dot{z}_{c}^{2}+\left(x_{c}-x\right) \ddot{x}_{c}+\left(y_{c}-y\right) \ddot{y}_{c}+\left(z_{c}-z\right) \ddot{z}_{c} \\
G_{i}= & \dot{x}_{c}^{2}+\dot{y}_{c}^{2}+\dot{z}_{c}^{2}+\ldots \\
& +\left(x_{c}-X_{i}\right) \ddot{x}_{c}+\left(y_{c}-Y_{i}\right) \ddot{y}_{c}+\left(z_{c}-Z_{i}\right) \ddot{z}_{c}
\end{aligned}
$$

and $\left(\ddot{x}_{c}, \ddot{y}_{c}, \ddot{z}_{c}\right)$ denotes the second-order temporal derivatives of $\left(x_{a}, y_{a}, z_{a}\right)$ at $t=0$.

Similarly, the detailed derivations of (19) can refer to [17]. By restricting $\Phi_{q i}(x, y)$ to a certain threshold value $\eta$, a DeR can be defined. When the ROI exceeds the DeR, we can solve this problem through a division-imaging-stitch way. Similarly, for a flat scenario, we have $\Phi_{q i 0}(x, y)=\Phi_{q i}(x, y ; z=0$, $\left.Z_{i}=0\right)$.

\section{Brief Summary and Outlook}

As OPFA inherits all the advantages of RZPFA, it is an efficient, distortion-free, defocus-negligible, zoom-able, and persistent imaging algorithm for spotlight SAR imaging under curvilinear trajectory. Since RZPFA only considered the distortion caused by plane-wave assumption in a flat scene, it can only achieve quasi-orthorectified imaging [17]. While for OPFA, with DEM distortion incorporated naturally in the built-in distortion compensation of RZPFA, orthorectified imaging becomes possible via a similar NuFFT-3. As the differences of the two algorithms lie only in the items related to $z$ and $Z_{i}$, their complexities are very close. After filling the gap of orthorectified imaging, now OPFA has realized all the advantages of $\mathrm{BP}$, yet it is much more efficient, providing a favorable choice for general spotlight SAR imaging.

As a byproduct of this algorithm, when DEM and the flight trajectory are both known, the derived mappings can be used to orthorectify PFA or BP images that were formed without DEM via image interpolation [14], [15]. Vice versa, when DEM is not given, then the derived functions also provide accurate mappings for DEM extraction from multiview SAR images [23], [24]. Furthermore, the proposed algorithm can be further modified in several ways: 1) utilized as an efficient forward and adjoint operator for iterative SAR imaging and simultaneous autofocusing via solving an inverse problem [25], [26];2) adapted to enable and accelerate large scene imaging by leveraging the division-decimation-upsamplingrecursion technique like fast BP algorithms [27]-[29]; and 3 ) extend the OPFA to advanced SAR configurations such as bistatic PFA [30], [31].

\section{EXPERIMENTS AND DISCUSSION}

\section{A. Data Preparation}

Regarding the radar echo data, the open data set named Gotcha large scene data was utilized [32]. The data set was collected in spotlight mode with a bandwidth of $600 \mathrm{MHz}$ centered at $9.6 \mathrm{GHz}$, illuminating a scene of about $5 \mathrm{~km} \times 5 \mathrm{~km}$. The data collection consists of ten successive blocks along slow time, each block has 3000 pulses, and each pulse has 21232 range samples. 
For the DEM data, we downloaded one that covers the Gotcha data area from the United States Geological Survey (USGS) website [33]. It is a 3-D elevation program seamless product with a resolution of $1 / 3$ arc-second and coverage of 1 square degree. The whole latitude ranges from $39^{\circ} \mathrm{N}$ to $40^{\circ} \mathrm{N}$, and longitude ranges from $85^{\circ} \mathrm{W}$ to $84^{\circ} \mathrm{W}$.

Let $\phi_{\text {lat } 0}, \phi_{\text {lon } 0}$, and $Z_{0}$ denote the latitude, longitude, and height of the reference center o. For a maximum extent of $5 \mathrm{~km} \times 5 \mathrm{~km}$, the earth curvature is trivial, thus can be ignored, and (latitude, longitude) can be regarded as linearly related to the $(x, y)$ since the OCS is a local east-north-up coordinate system. According to an approximate coordinates transform from Wikipedia [34], we have

$$
\begin{aligned}
\text { Lat }_{1}= & 111132.92-559.82 \times \cos \left(2 \phi_{\text {lat } 0}\right)+\ldots \\
& +1.175 \times \cos \left(4 \phi_{\text {lat } 0}\right)-0.0023 \times \cos \left(6 \phi_{\text {lat } 0}\right) \\
\text { Lon }_{1}= & 111412.84 \times \cos \left(\phi_{\text {lon } 0}\right)+\ldots \\
& -93.5 \times \cos \left(3 \phi_{\text {lon } 0}\right)+0.118 \times \cos \left(5 \phi_{\text {lon } 0}\right)
\end{aligned}
$$

where Lat ${ }_{1}$ and $\mathrm{Lon}_{1}$ are the ground length for one degree in latitude and longitude at $\mathbf{o}$.

For each pixel of the ROI grid $\left(x_{r}, y_{r}\right)$ in OCS, its corresponding latitude and longitude can be approximated as

$$
\phi_{\text {lat }}\left(y_{r}\right)=\frac{y_{r}}{\text { Lat }_{1}}+\phi_{\text {lat } 0}, \quad \phi_{\text {lon }}\left(x_{r}\right)=\frac{x_{r}}{\operatorname{Lon}_{1}}+\phi_{\text {lon } 0} .
$$

As the coordinates of the reference point are not given in the Gotcha data, we calibrated the values based on the imaging quality of BP and OPFA, and the resulting values are $\phi_{\text {lat0 }}=$ $39.779721^{\circ} \mathrm{N}, \phi_{\text {lon } 0}=84.098365^{\circ} \mathrm{W}, Z_{0}=244 \mathrm{~m}, \mathrm{Lat}_{1}=$ $110992.3 \mathrm{~m}$, and Lon $_{1}=11484.1 \mathrm{~m}$. With the above equations and values, for any ROI grid within the Gotcha data area, we can obtain its DEM via 2-D interpolation on the original DEM. After that, a subtraction of $Z_{0}$ is conducted to get the final DEM defined in OCS.

\section{B. Imaging Results of a Large ROI}

First, we tested the imaging performance of OPFA for a large centered ROI with an extent of $4096 \mathrm{~m} \times 4096 \mathrm{~m}$, with RZPFA as a benchmark. Only the first five blocks of the Gotcha data were used because of the limited memory. The obtained DEM is shown in Fig. 2(a), and after subtracting $Z_{0}$, the maximum and minimum heights within the ROI are 64.52 and $-14.37 \mathrm{~m}$, respectively, with an average value of $13.26 \mathrm{~m}$. Calculated according to (18), the DEM distortion without refocusing is shown in Fig. 2(b). The DEM distortion values show a positive correlation with the DEM values in Fig. 2(a), and both present a general increasing trend from left to right and from top to bottom. Within the large ROI, the maximum and average displacements caused by DEM distortion are 75.54 and $17.96 \mathrm{~m}$, which could not be neglected for demanding applications.

Although it is straightforward to compensate for the residual distortion after imaging via image interpolation with the derived distortion mappings, we will show that it is unnecessary since we could incorporate all the necessary distortion in the OPFA imaging process. Unless otherwise specified, the fast Gaussian grid nonuniform fast Fourier transform (FGG-NuFFT) [18], [35] was used for RZPFA and OPFA with

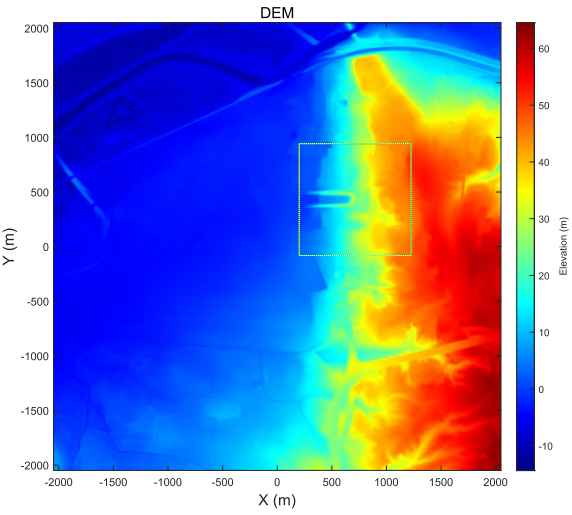

(a)

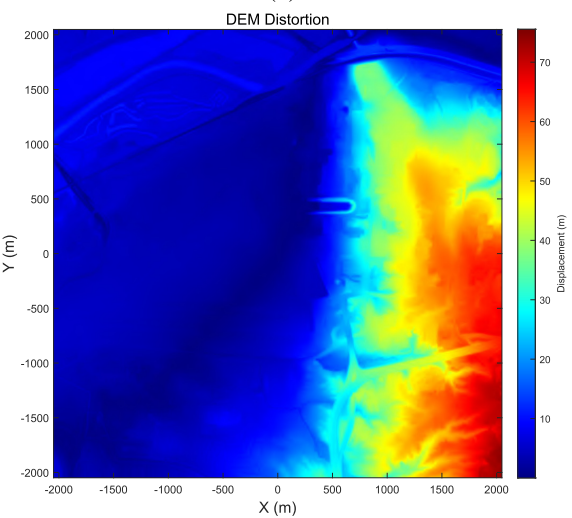

(b)

Fig. 2. (a) DEM of the large ROI. (b) DEM distortion of the large ROI.

an error threshold of $10^{-6}$, and all imaging results are shown in normalized magnitude above $-70 \mathrm{~dB}$.

For the large ROI, the results via RZPFA and OPFA are shown in Fig. 3. Both figures were formed without postprocessing, with most streets being straight and most buildings being rectangular. However, if we have a close-up view of the bottom right part, there is an obvious displacement between the two figures, which represents the residual DEM distortion and attributes to the large displacement values shown in Fig. 2(b).

Furthermore, the approximated maximum RQPE without $\operatorname{DEM}\left(\Phi_{q i 0}\right)$ and with DEM $\left(\Phi_{q i}\right)$ were calculated and shown by a green contour $(\eta=\pi / 8)$ and a magenta contour ( $\eta=\pi / 4)$ in Fig. 3(a) and (b). It is shown that the contours in Fig. 3(a) are very smooth when DEM is not considered. While in Fig. 3(b), the contours tend to bend in nonflat areas. By comparing these two figures, the defocus of a certain area, such as the closed inner contour in the top right of Fig. 3(b), becomes nonnegligible just because of the large elevation offsets from the imaging plane. An interesting phenomenon can be observed in the center of the white frame in Fig. 3(b), and the contours, in fact, form a U-pattern, showing accordance with the U-pattern terrain bump in Fig. 2.

\section{Imaging Results of Smaller ROIs}

To better illustrate the effect of OPFA for residual DEM distortion compensation, the bottom right quarter of the large ROI was imaged using RZPFA and OPFA, and the orthoimagery 


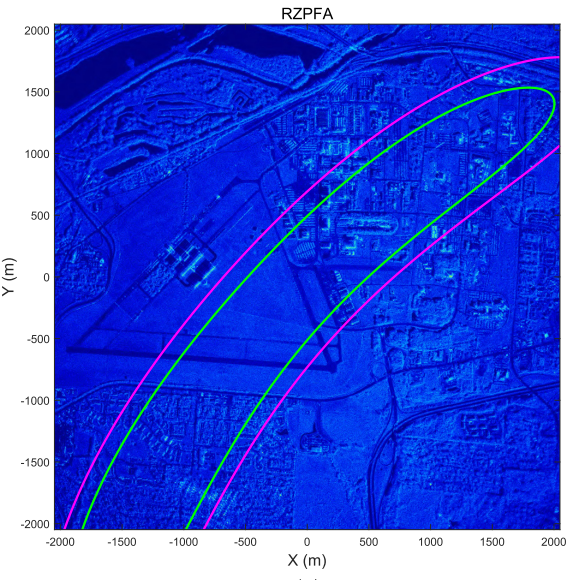

(a)

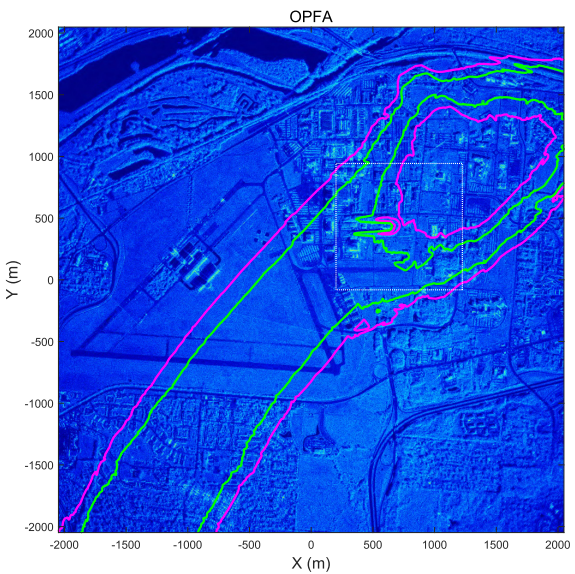

(b)

Fig. 3. Imaging results of the large ROI. (a) RZPFA. (b) OPFA.

from Google Earth was chosen as a comparison. The results are shown in Fig. 4, and the reader is kindly requested to ignore changes such as new buildings as the orthoimagery was collected later than the Gotcha data. Fig. 4(a) and (d) shows the results formed by RZPFA and OPFA, respectively. To facilitate comparison, the same orthoimagery is presented twice in Fig. 4(b) and (c), and some auxiliary straight lines are drawn. After the refocusing on the center of the smaller ROI, the residual defocus can be ignored, and both Fig. 4(a) and (d) are well-focused. However, by comparing the RZPFA image with the orthoimagery horizontally and vertically, notable distortion can be observed, which demonstrates the existence of residual DEM distortion. While for the OPFA image, all features are well aligned with the corresponding features in the orthoimagery, which validated its orthorectified imaging capability. To further facilitate comparison, the top right portions of Fig. 4 are zoomed and shown in Fig. 5.

Finally, the U-pattern area within the white frame in Fig. 2(a) is formed via RZPFA, OPFA, BP (with DEM), and BP (without DEM). For RZPFA and OPFA, based on the calculations of approximate maximum RQPE via (19), defocus can be ignored after the refocusing. At this time, to reduce the imaging time of BP, only one block of Gotcha data was utilized, and the BP code in [36] was implemented. The average elapsed time in seconds over the ten blocks for
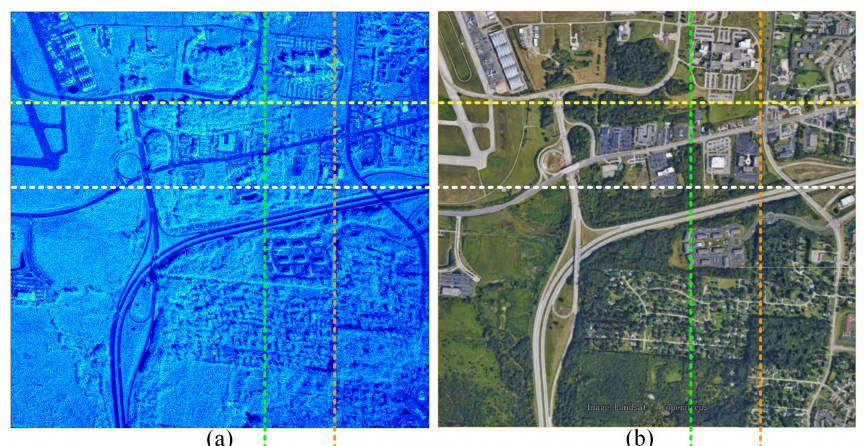

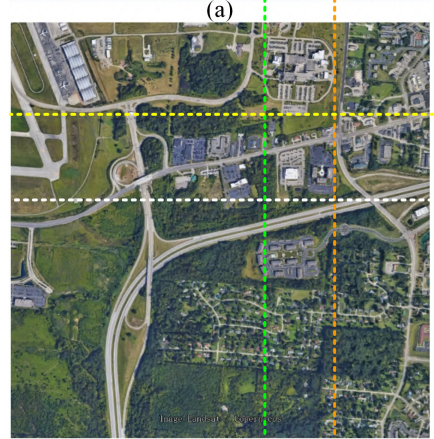

(c)

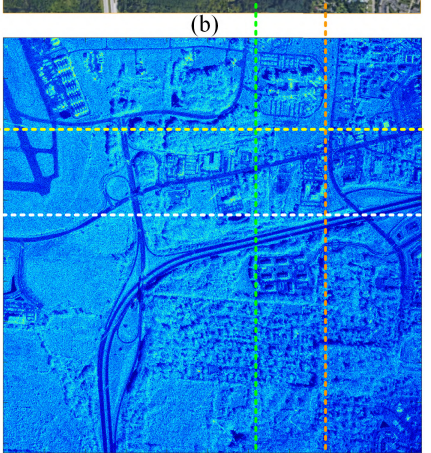

(d)
Fig. 4. Imaging results of the bottom right quarter ROI. (a) RZPFA. (b) and (c) Google Earth orthoimagery. (d) OPFA

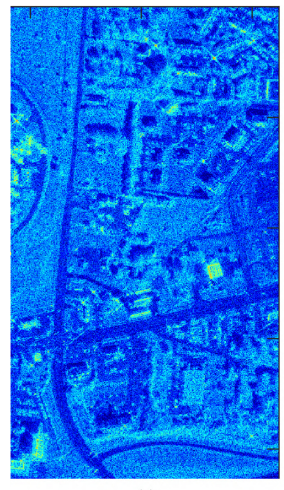

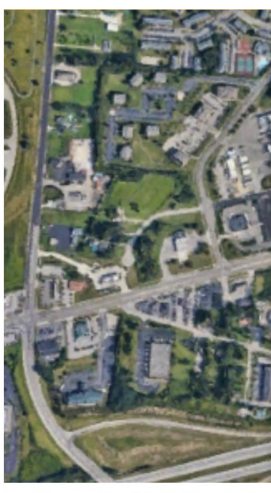

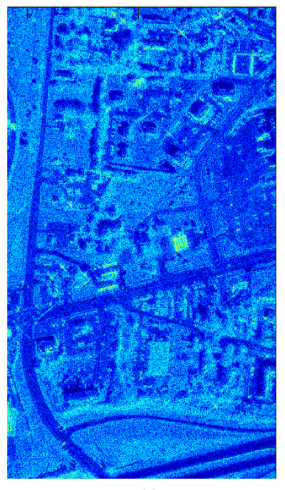

Fig. 5. Zoomed-in results of the top right portions of Fig. 4. (a) RZPFA. (b) Google earth orthoimagery. (c) OPFA.

TABLE I

AVERAGE Elapsed TIME COMPARISON

\begin{tabular}{|c|c|c|c|c|}
\hline & BP (w/o DEM) & BP (w/ DEM) & RZPFA & OPFA \\
\hline $512 \times 512$ & $169.5 \mathrm{~s}$ & $171.5 \mathrm{~s}$ & $75.8 \mathrm{~s}$ & $77.2 \mathrm{~s}$ \\
\hline $1024 \times 1024$ & $613.2 \mathrm{~s}$ & $642.4 \mathrm{~s}$ & $77.1 \mathrm{~s}$ & $78.7 \mathrm{~s}$ \\
\hline
\end{tabular}

pixel numbers of $512 \times 512$ and $1024 \times 1024$ was recorded and is shown in Table I. The time values show that RZPFA and OPFA are much efficient than BP. When the number of pixel grew from $512 \times 512$ to $1024 \times 1024$, the elapsed time of BP grew dramatically, whereas RZPFA and OPFA only experienced a very small increase. Besides, the extra time of OPFA over RZPFA is very small, which validated the previous analysis. 


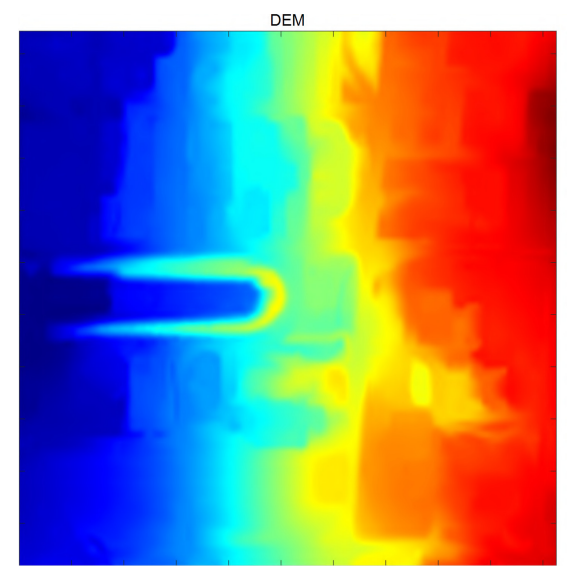

(a)

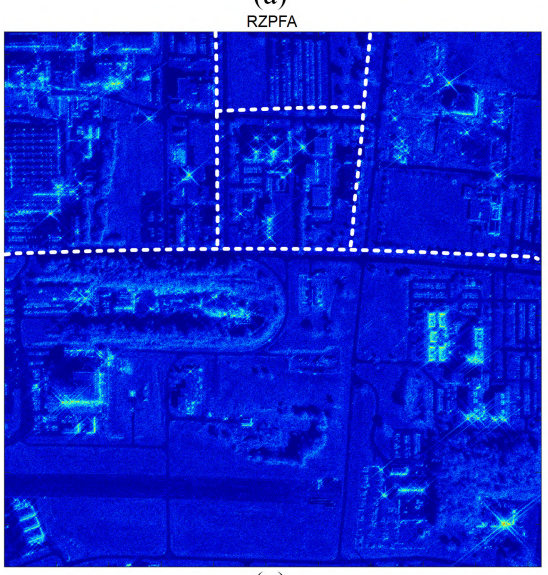

(c)

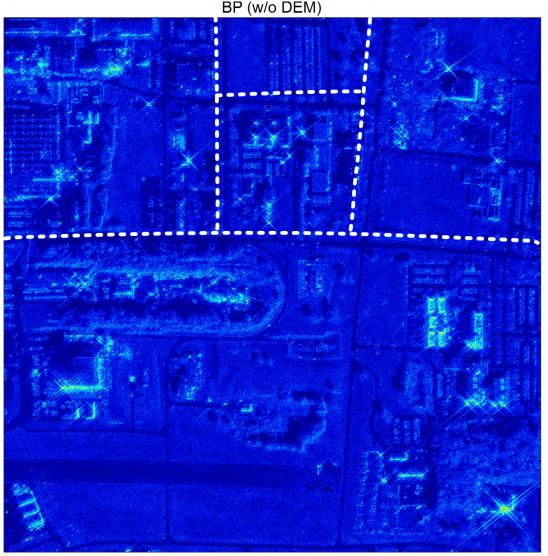

(e)

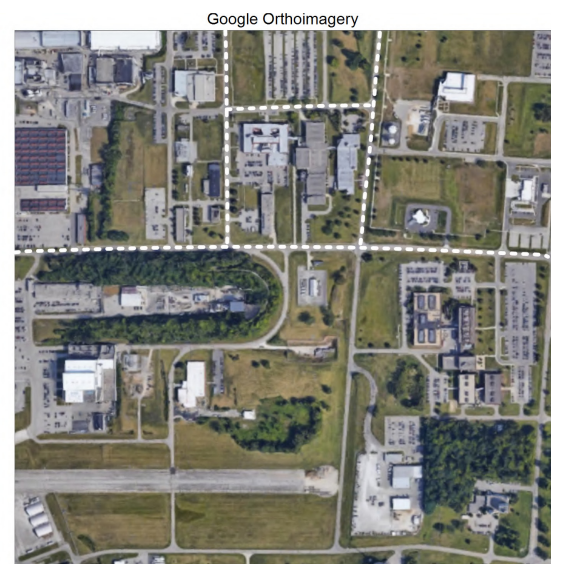

(b)

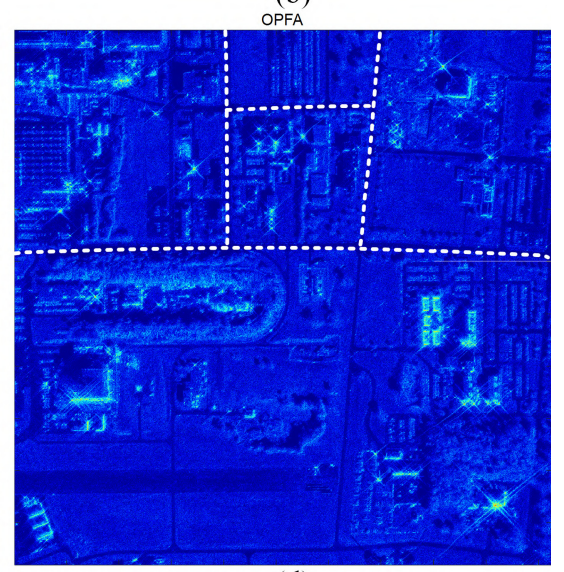

$(\mathrm{d})$

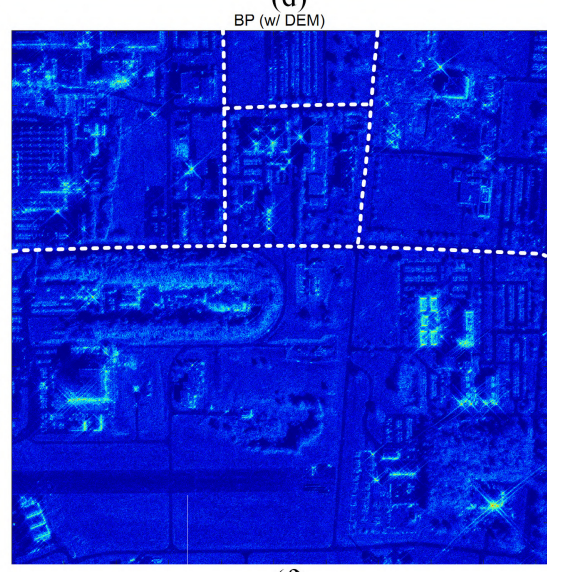

(f)

Fig. 6. Imaging results of the U-pattern area. (a) DEM. (b) Google Earth orthoimagery. (c) RZPFA. (d) OPFA. (e) BP (without DEM). (f) BP (with DEM).

The imaging results of the last data block are shown in Fig. 6, where Fig. 6(a) and (b) shows the DEM and orthoimagery of the U-pattern area, respectively. Fig. 6(c) and (d) shows the results via RZPFA and OPFA. Fig. 6(e) and (f) shows the results via BP without and with DEM. For an easier observation, some roads in Fig. 6(b) were extracted and represented by white dotted lines and then were superimposed on the same location in Fig. 6(c)-(f). In Fig. 6(c) and (e), the white dotted lines could not cover the corresponding roads, showing an evident residual distortion for RZPFA and BP (without DEM). Nevertheless, their same distortion pattern
TABLE II

Correlation Values Between Image Pairs

\begin{tabular}{|c|c|c|c|c|}
\hline & OPFA & RZPFA & BP (w/ DEM) & BP (w/o DEM) \\
\hline OPFA & $/$ & 0.0926 & $\mathbf{0 . 9 9 5 5}$ & 0.0934 \\
\hline RZPFA & 0.0926 & $/$ & 0.0919 & $\mathbf{0 . 9 9 6 4}$ \\
\hline
\end{tabular}

proved the equivalence between them. On the contrary, the dotted lines matched with the roads in Fig. 6(d) and (f) perfectly, so as other feathers demonstrated the orthorectified imaging 
ability of OPFA and also an equivalence between OPFA and BP (with DEM).

The correlation value between magnitude image pairs for the above four imaging methods was calculated and is shown in Table II. The extremely high correlation values between RZPFA and BP (without DEM) and between OPFA and BP (with DEM) also support the conclusions numerically.

\section{CONCLUSION}

In this article, OPFA for general curvilinear spotlight SAR orthorectified imaging on arbitrary ROI with DEM is proposed. Through OPFA, orthorectified images can be formed efficiently without any postprocessing. OPFA has comparable performance and flexibility as BP and is more efficient than BP, making it as a competitive choice for spotlight SAR imaging. The effectiveness of the proposed method has been verified by the results of real data.

\section{ACKNOWLEDGMENT}

The authors would like to thank Dingfan Xing for her helpful advice on geographic coordinate systems.

\section{REFERENCES}

[1] C. Toth and G. Jóków, "Remote sensing platforms and sensors: A survey," ISPRS J. Photogramm. Remote Sens., vol. 115, pp. 22-36, May 2016.

[2] A. Moreira, P. Prats-Iraola, M. Younis, G. Krieger, I. Hajnsek, and K. P. Papathanassiou, "A tutorial on synthetic aperture radar," IEEE Geosci. Remote Sens. Mag., vol. 1, no. 1, pp. 6-43, Mar. 2013.

[3] X. X. Zhu et al., "Deep learning in remote sensing: A comprehensive review and list of resources," IEEE Geosci. Remote Sens. Mag., vol. 5, no. 4, pp. 8-36, Dec. 2017.

[4] P. Laurila, R. Modrzewski, T. Cheng, B. Campbell, and V. G. Yanni, "Validation of ICEYE small satellite SAR design for ice detection and imaging," in Proc. Arctic Technol. Conf., 2016, p. 5.

[5] G. Farquharson, W. Woods, C. Stringham, N. Sankarambadi, and L. Riggi, "The capella synthetic aperture radar constellation," in Proc. IEEE Int. Geosci. Remote Sens. Symp., Jul. 2018, pp. 1-5.

[6] W. Pu et al., "Motion errors and compensation for bistatic forwardlooking SAR with cubic-order processing," IEEE Trans. Geosci. Remote Sens., vol. 54, no. 12, pp. 6940-6957, Dec. 2016.

[7] G. Krieger and A. Moreira, "Spaceborne bi-and multistatic SAR: Potential and challenges," IEE Proc. Radar, Sonar Navig., vol. 153, no. 3, pp. 184-198, 2006.

[8] D. Cerutti-Maori, I. Sikaneta, J. Klare, and C. H. Gierull, "MIMO SAR processing for multichannel high-resolution wide-swath radars," IEEE Trans. Geosci. Remote Sens., vol. 52, no. 8, pp. 5034-5055, Aug. 2014.

[9] R. Hu, R. Min, and Y. Pi, "A video-SAR imaging technique for aspectdependent scattering in wide angle," IEEE Sensors J., vol. 17, no. 12, pp. 3677-3688, Jun. 2017.

[10] W. Pu, X. Wang, J. Wu, Y. Huang, and J. Yang, "Video SAR imaging based on low-rank tensor recovery," IEEE Trans. Neural Netw. Learn. Syst., early access, Jun. 16, 2020, doi: 10.1109/TNNLS.2020. 2978017.

[11] W. Wang, D. An, Y. Luo, and Z. Zhou, "The fundamental trajectory reconstruction results of ground moving target from single-channel CSAR geometry," IEEE Trans. Geosci. Remote Sens., vol. 56, no. 10, pp. 5647-5657, Oct. 2018.

[12] N. Ö. Onhon and M. Cetin, "A sparsity-driven approach for joint SAR imaging and phase error correction," IEEE Trans. Image Process., vol. 21, no. 4, pp. 2075-2088, Apr. 2012.

[13] J. Walker, "Range-Doppler imaging of rotating objects," IEEE Trans. Aerosp. Electron. Syst., vol. AES-16, no. 1, pp. 23-52, Jan. 1980.
[14] M. Shimada, "Ortho-rectification and slope correction of SAR data using DEM and its accuracy evaluation," IEEE J. Sel. Topics Appl. Earth Observ. Remote Sens., vol. 3, no. 4, pp. 657-671, Dec. 2010.

[15] Y. Sheng and D. E. Alsdorf, "Automated georeferencing and orthorectification of amazon basin-wide SAR mosaics using SRTM DEM data," IEEE Trans. Geosci. Remote Sens., vol. 43, no. 8, pp. 1929-1940, Aug. 2005.

[16] C. V. Jakowatz, Jr., D. E. Wahl, and D. A. Yocky, "Beamforming as a foundation for spotlight-mode SAR image formation by backprojection," Proc. SPIE-Algorithms Synth. Aperture Radar Imag., vol. 6970, Mar. 2008, Art. no. 69700Q

[17] R. Hu et al., "Refocusing and zoom-in polar format algorithm for curvilinear spotlight SAR imaging on arbitrary region of interest," IEEE Trans. Geosci. Remote Sens., vol. 57, no. 10, pp. 7995-8010, Oct. 2019.

[18] J.-Y. Lee and L. Greengard, "The type 3 nonuniform FFT and its applications," J. Comput. Phys., vol. 206, no. 1, pp. 1-5, Jun. 2005.

[19] A. H. Barnett, J. Magland, and L. af Klinteberg, "A parallel nonuniform fast Fourier transform library based on an exponential of semicircle' kernel," SIAM J. Sci. Comput., vol. 41, no. 5, pp. 479-504, Jan. 2019.

[20] L. A. Gorham and B. D. Rigling, "Scene size limits for polar format algorithm," IEEE Trans. Aerosp. Electron. Syst., vol. 52, no. 1, pp. 73-84, Feb. 2016.

[21] L. Gorham and B. Rigling, "Fast corrections for polar format algorithm with a curved flight path," IEEE Trans. Aerosp. Electron. Syst., vol. 52, no. 6, pp. 2815-2824, Dec. 2016.

[22] R. Hu, F. Zuo, X. Li, X. Hu, Y. T. Soon, and C. Ma, "Curvilinear videosar persistent imaging with distortion correction based on NUFFT-3," in Proc. IEEE Int. Geosci. Remote Sens. Symp., Aug. 2019, pp. 684-687.

[23] U. Soergel, E. Michaelsen, A. Thiele, E. Cadario, and U. Thoennessen, "Stereo analysis of high-resolution SAR images for building height estimation in cases of orthogonal aspect directions," ISPRS J. Photogramm. Remote Sens., vol. 64, no. 5, pp. 490-500, Sep. 2009.

[24] S. Palm, H. M. Oriot, and H. M. Cantalloube, "Radargrammetric DEM extraction over urban area using circular SAR imagery," IEEE Trans. Geosci. Remote Sens., vol. 50, no. 11, pp. 4720-4725, Nov. 2012.

[25] S. Matej, J. A. Fessler, and I. G. Kazantsev, "Iterative tomographic image reconstruction using Fourier-based forward and back-projectors," IEEE Trans. Med. Imag., vol. 23, no. 4, pp. 401-412, Apr. 2004.

[26] S. I. Kelly, G. Rilling, M. Davies, and B. Mulgrew, "Iterative image formation using fast (re/back)-projection for spotlight-mode SAR," in Proc. IEEE Radar Conf., May 2011, pp. 835-840.

[27] S. I. Kelly and M. E. Davies, "A fast decimation-in-image back-projection algorithm for SAR," in Proc. Radar Conf., 2014, pp. 1046-1051

[28] L. M. H. Ulander, H. Hellsten, and G. Stenstrom, "Synthetic-aperture radar processing using fast factorized back-projection," IEEE Trans. Aerosp. Electron. Syst., vol. 39, no. 3, pp. 760-776, Jul. 2003.

[29] J. W. McCorkle and M. Rofheart, "Order nर̂ $\log$ (n) backprojector algorithm for focusing wide-angle wide-bandwidth arbitrary-motion synthetic aperture radar,' in Proc. Radar Sensor Technol., vol. 2747, 1996, pp. 25-36.

[30] D. Mao and B. D. Rigling, "Distortion correction and scene size limits for SAR bistatic polar format algorithm," in Proc. IEEE Radar Conf. (RadarConf), May 2017, pp. 1103-1108.

[31] Q. Zhang, J. Wu, Z. Li, Y. Miao, Y. Huang, and J. Yang, "PFA for bistatic forward-looking SAR mounted on high-speed maneuvering platforms," IEEE Trans. Geosci. Remote Sens., vol. 57, no. 8, pp. 6018-6036, Aug. 2019.

[32] Air Force Research Laboratory. Gotcha large scene data. Accessed: Jul. 8, 2020. [Online]. Available: https://www.sdms.afrl.af.mil

[33] U.S. Geological Survey. Usgs 13 Arc-Second n40w085 $1 \times 1$ Degree. Accessed: Jul. 8, 2020. [Online]. Available: https://www.sciencebase. gov/catalog/item/5deb329ae4b02caea0f0ea8f

[34] Wikipedia contributors. Geographic Coordinate System-Wikipedia the Free Encyclopedia. Accessed: Jul. 8, 2020. [Online]. Available: https://en.wikipedia.org/w/index.php?title=Geographic_coordinate _system\&oldid $=954936865$

[35] L. Greengard and J.-Y. Lee, "Accelerating the nonuniform fast Fourier transform," SIAM Rev., vol. 46, no. 3, pp. 443-454, Jan. 2004.

[36] L. A. Gorham and L. J. Moore, "SAR image formation toolbox for MATLAB," Proc. SPIE Algorithms Synth. Aperture Radar Imag., vol. 7699, Dec. 2010, Art. no. 769906 


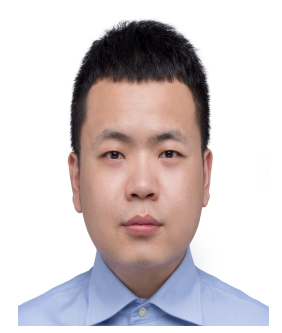

Ruizhi Hu (Member, IEEE) received the B.S. and $\mathrm{Ph} . \mathrm{D}$. degrees from the University of Electronic Science and Technology of China (UESTC), Chengdu, China, in 2012 and 2018, respectively.

He was a Research Fellow with the Department of Electrical and Computer Engineering, National University of Singapore, Singapore, from 2018 to 2019. He joined SIGCOM Group, Interdisciplinary Centre for Security, Reliability and Trust (SnT), University of Luxembourg, Luxembourg City, Luxembourg, in March 2020, as a Research Associate. His research interests lie in the area of SAR imaging, automotive radar signal processing, and machine learning.

Dr. $\mathrm{Hu}$ is a Reviewer of the IEEE TRANSACTIONS ON GEOSCIENCE AND Remote Sensing and the IEEE Geoscience and Remote Sensing LETTERS.

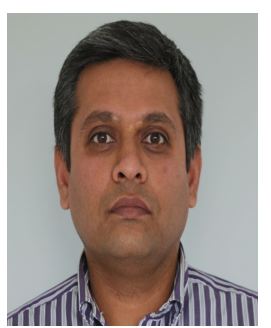

Bhavani Shankar Mysore Rama Rao (Senior Member, IEEE) received the master's and Ph.D. degrees in electrical communication engineering from the Indian Institute of Science, Bengaluru, India, in 2000 and 2007, respectively.

He held a post-doctoral position at ACCESS Linnaeus Centre, Signal Processing Laboratory, KTH Royal Institute of Technology, Stockholm, Sweden, from 2007 to September 2009. He joined SnT, University of Luxembourg, Luxembourg City, Luxembourg, in October 2009, as a Research Associate, where he is a Research Scientist. He was with Beceem Communications, Bengaluru, India, from 2006 to 2007, as a Staff Design Engineer working on physical layer algorithms for WiMAX compliant chipsets. He was a Visiting Student at Communication Theory Group, ETH Zürich, Zürich, Switzerland, headed by Prof. Helmut Bölcskei in 2004. Prior to joining Ph.D., he worked on audio coding algorithms in Sasken Communications, Bengaluru, from 2000 to 2001, as a Design Engineer. His research interests include design and optimization of MIMO communication systems, automotive radar and array processing, polynomial signal processing, satellite communication systems, resource allocation, game theory, and fast algorithms for structured matrices.

Dr. Rao is on the Executive Committee of the IEEE Benelux Joint Chapter on Communications and Vehicular Technology, a member of the EURASIP Technical Area Committee on Theoretical and Methodological Trends in Signal Processing, and serves as a Handling Editor for Signal Processing (Elsevier). He was a co-recipient of the 2014 Distinguished Contributions to Satellite Communications Award from the Satellite and Space Communications Technical Committee of the IEEE Communications Society.

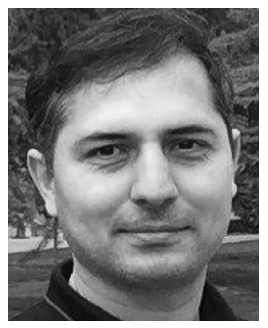

Mohammad Alaee-Kerahroodi (Member, IEEE) received the Ph.D. degree in telecommunication engineering from the Department of Electrical and Computer Engineering, Isfahan University of Technology, Isfahan, Iran.

From February to August 2016, he was a Visiting Researcher with the University of Naples "Federico II," Naples, Italy. In 2017, he joined SIGCOM Group, Interdisciplinary Centre for Security, Reliability and Trust (SnT), University of Luxembourg, Luxembourg City, Luxembourg. He is working toward innovative radar signal processing solutions for automotive MIMO radar systems as well as pursuing academic research in the area of radar waveform design and signal processing. His research interests are related to waveform design for active sensing and communication systems, optimization theory, and statistical/array signal processing.

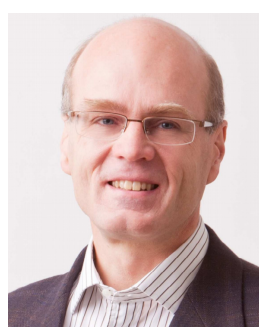

Björn Ottersten (Fellow, IEEE) was born in Stockholm, Sweden, in 1961. He received the M.S. degree in electrical engineering and applied physics from Linköping University, Linköping, Sweden, in 1986, and the Ph.D. degree in electrical engineering from Stanford University, Stanford, CA, USA, in 1990.

$\mathrm{He}$ has held research positions with the Department of Electrical Engineering, Linköping University, Information Systems Laboratory, Stanford University, the Katholieke Universiteit Leuven, Leuven, Belgium, and the University of Luxembourg, Luxembourg City, Luxembourg. From 1996 to 1997, he was the Director of Research with ArrayComm, Inc., a startup in San Jose, CA, USA, based on his patented technology. In 1991, he was appointed as a Professor of signal processing with the Royal Institute of Technology (KTH), Stockholm. He has been the Head of the Department for Signals, Sensors, and Systems, KTH, and the Dean of the School of Electrical Engineering, KTH. He is the Director of the Interdisciplinary Centre for Security, Reliability and Trust, University of Luxembourg.

Dr. Ottersten is a fellow of EURASIP. He was a recipient of the IEEE Signal Processing Society Technical Achievement Award and the European Research Council Advanced Research Grant twice. He has coauthored journal articles that received the IEEE Signal Processing Society Best Paper Award in 1993, 2001, 2006, 2013, and 2019, and eight IEEE conference papers' best paper awards. He has been a Board Member of the IEEE Signal Processing Society, the Swedish Research Council, and serves on the boards of EURASIP and the Swedish Foundation for Strategic Research. He has served as an Associate Editor for the IEEE TRANSACTIONS ON SIGNAL PROCESSING and the Editorial Board of the IEEE Signal Processing Magazine. $\mathrm{He}$ is a member of the editorial boards of the IEEE OPEN Journal of Signal Processing, the EURASIP Signal Processing Journal, the EURASIP Journal of Advances Signal Processing, and the Foundations and Trends in Signal Processing. 